\title{
Damage evaluation based on ultrasonic testing of compos-ite aircraft elements and image analysis methods
}

\author{
Angelika Wronkowicz ${ }^{1, *}$ and Krzysztof Dragan ${ }^{2}$ \\ ${ }^{1}$ Silesian University of Technology, Institute of Fundamentals of Machinery Design, Konarskiego \\ 18A, 44-100 Gliwice, Poland \\ ${ }^{2}$ Air Force Institute of Technology, Laboratory of Non-Destructive Testing, 6 Ks. Bolesława, 01-494 \\ Warsaw, Poland
}

\begin{abstract}
Composite aircraft elements are inspected with the use of nondestructive testing methods to detect the damage appearance and monitor its progression. Ultrasonic testing is one of the most commonly applied methods owing to its capabilities of detecting various types of internal flaws and dam-age as well as evaluating their location and geometrical properties. There are several di culties in analysing the ultrasonic data, which cover the high time-consumption of such analyses resulting from large sizes of the scans and a man-ual handling of them. Thus, due to the possible occurrence of human errors, there is a necessity of increasing the e ciency of these inspections by aiding the analysis of the ultrasonic data. The paper presents proposed methods of the application of image analysis methods for supporting the procedures of the ul-trasonic evaluation of the aircraft structures. These cover facilitating the steps of damage detection, quantification as well as a three dimensional reconstruc-tion and visualisation. The proposed approaches are computationally e cient and may facilitate the data interpretation and damage evaluation procedures.
\end{abstract}

\section{Introduction}

Polymeric composite materials are nowadays widely applied for manufacturing of structural components of aircrafts. However, they are vulnerable to the formation of various types of defects during their production (e.g. porosity, foreign object inclusions) and damage during operation (e.g. delamination, matrix cracks, impact damage). Damage occurring in com-posite materials is a complex phenomenon and, under load, may propagate and interact with manufacturing defects up to failure. Because of that, composites are inspected with the use of non-destructive testing (NDT) methods to detect the damage appearance and monitor its progression. The maintenance procedures of aircraft elements are accomplished according to one of developed to-date design methodologies. Historically, the development of these methodologies was motivated by several tragic accidents in the aircraft industry [1] and the necessity of increasing safety and prolongation

* Corresponding author: angelika.wronkowicz@polsl.pl 
of airworthiness. One of the currently used approaches is based on so-called damage tolerance methodology, in which the existence of small cracks and defects in the aircraft structure is permissible if they can resist failure before the scheduled inspection. Therefore, one of the most important tasks in this methodology is to perform periodic non-destructive inspections of the critical aircraft elements.

\subsection{Ultrasonic inspections of structural aircraft elements}

An aircraft inspection can range from preflight and postflight inspections, which are a casual "walk-around" with a visual check for general condition and delivered mainly by the flight crew, to detailed inspections involving complete disassembly and the use of complex inspection aids and delivered by the qualified engineering sta . Among many NDT methods, ultrasonic testing (UT) is one of the most commonly applied [2] owing to its capabilities of detecting various types of damage and flaws inside composites, and evaluating their geomet-rical properties and location. Principally, the obtained output during such an inspection is in a form of ultrasonic data presenting levels of attenuation of the ultrasonic waves by particular areas of a tested structure. A change in that levels may indicate the presence of a defect or damage, but also noise or, for instance, an embedded element inside the structure, such as a sti ening rib or a rivet. The most commonly used mode of the data presentation for the damage evaluation purpose is a so-called ultrasonic CScan.

\subsection{Damage evaluation by post-processing of ultrasonic data}

Appropriate processing and analysis of ultrasonic data allow for the damage detection, locali-sation, characterisation, as well as quantification. By the separation of damage from a "back-ground" (i.e. the underlying healthy regions of the test structure) visible in the CScan, i.e. extraction of its contour, it is possible to calculate the damage extent. After the inspection, the obtained ultrasonic data is thoroughly analysed and a report with the description on the detected damage sites with annotations about their sizes and locations is prepared. In prac-tice, this is performed with the use of software dedicated to UT that usually provides only very simple tools for manual processing of the data. In the case of inspecting the structural aircraft elements such as vertical stabilizers that are very large the evaluation based on ul-trasonic data is time-consuming and, due to the need for manual parameter settings, human error is more likely to occur. There are several standardised methods employed for damage evaluation, i.e. quantification by the post-processing of the ultrasonic data, however, there is a need of verifying it by the application of the second method.

Various attempts can be found in the literature that are aimed at the application of image processing methods for aiding the analysis of ultrasonic scans. To the examples one can include the approaches employing wavelet transforms [3], feature extraction [4], fuzzy logic [5], data clustering [6], image segmentation based on statistical mean and standard devi-ation [7], or edge detection techniques [8]. Moreover, since interpretation of ultrasonic scans is sometimes di cult due to the presence of noise or the complexity of the structure geometry and the existence of embedded elements, their 3D visualisation could facilitate the diagnostic inference procedure. One of the approaches was presented in the study [9], where a 3D view was generated by means of a rule and threshold-based algorithm. A proposed method of the authors of this study is presented in [10]. 
However, these approaches were developed for specific problems and are not universal. The goal of the performed research was to increase e ciency and reliability of the currently implemented damage detection and evaluation procedures. Selected results obtained with the application of the developed image analysis algorithms are presented in the next sections.

\section{Structures and experiments}

The tested element considered in this study is a composite panel used in military aircrafts with delaminations formed during maintenance. As an exemplary structure, a part made of CFRP KMU-4e: ELUR-0.1p and ENFB based on epoxy resin (the stacking sequence of laminates' layers: $0,90,-45,+45$ ) was considered. The thickness of the inspected panel is equal to $4 \mathrm{~mm}$. A demonstrative fragment of this element is presented in Figure 1(a). In the tested panel, the delaminated areas developed around an inspection hatch of an elliptic shape, probably caused by over-tightening during assembly, as well as in the area of rivet holes.

The panel was tested using the MAUS ${ }^{\mathrm{R}}$ system with a $5 \mathrm{MHz}$ single transducer and a resolution of 0.01 ". A fragment of the obtained ultrasonic C-Scan is presented in Figure 1(b).

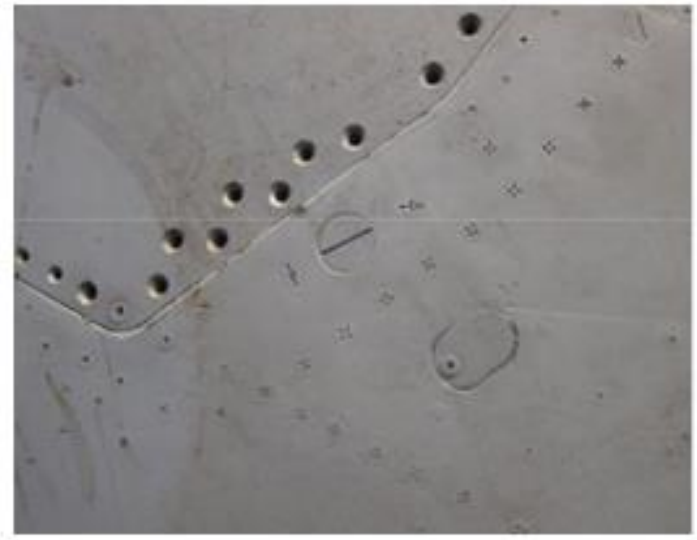

(a)

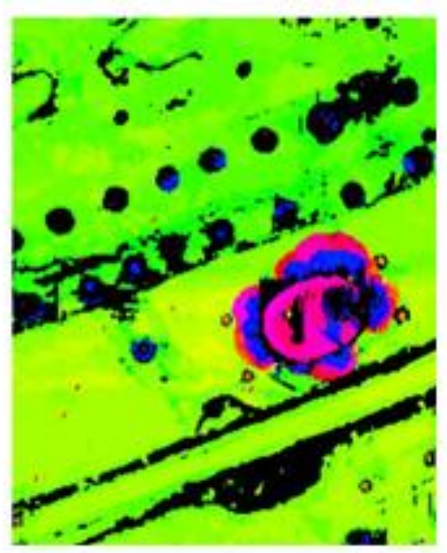

(b)

Fig. 1. A fragment of the aircraft element (a) and a fragment of ultrasonic C-Scan (b) [11]

\section{Selected image analysis algorithms}

\subsection{Damage detection}

In order to calculate the extent of damage observed in the ultrasonic scan it is necessary to firstly detect it, i.e. separate the pixels representing damage from other pixel regions visible in the C-Scan. The key aspect in the damage detection is proper image segmentation, i.e. data reduction, which allows for a quicker and easier separation of the damage from the background ultrasonic reflections. 


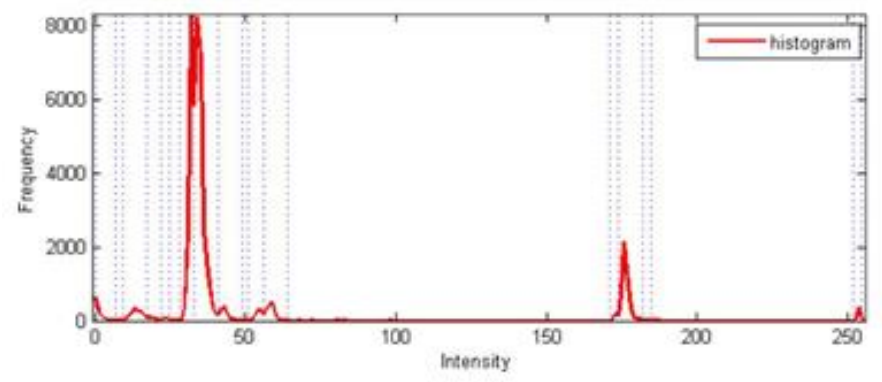

(a)

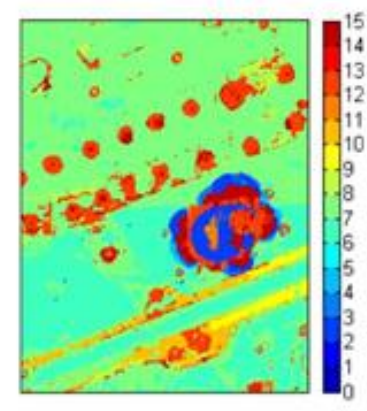

(b)

Fig. 2. Histogram of the C-Scan and the image after the segmentation [11]

The proposed method is called a multilevel segmentation using a Minima-BetweenPeaks (MBP) criterion, and was described in detail in [11]. This consists in a selection of all the local minima of the image histogram as the thresholds by which the image is divided into segments. A histogram of the considered C-Scan is presented in Figure 2(a), where the dotted lines show locations of the obtained thresholds. The resulting image after the segmentation is presented in Figure 2(b), where one can also observe the resulting numbers of classes. After that, the regions of interests (ROIs) can be easily extracted by the selection of the segments that are to be left in (or removed from) the image.

\subsection{Damage detection}

The second case study was dedicated to delamination size monitoring based on pairs of CScans acquired during UT carried out in two-year inspection intervals during the aircraft operation. Initial results of the research in this area were published in [12].

The proposed algorithm is divided into two main stages: damage extraction and quantifi-cation (which is performed for both input C-Scans separately) and damage growth calculation and comparison (where the results of the first stage are analysed together). Firstly, the input image is segmented using the MBP-based algorithm (see the exemplary result in Figure 3(a)). Then, segments of the healthy regions are removed from the image (Figure 3(b)). The next step is the interactive ROI selection (Figure 3(c)) in order to exclude the regions of the embedded elements in the structure (e.g. not removed rivets). Additional processing based on morpho-logical operations is performed in order to clean possibly remaining pixels. The resulting image is presented in Figure 3(d). Finally, boundaries of the resulting object are extracted and its surface area is calculated. The resulting images with the extracted boundaries (in red) are presented in Figure 4(a) and Figure 4(b), respectively, for the exemplary pair of C-Scans. 


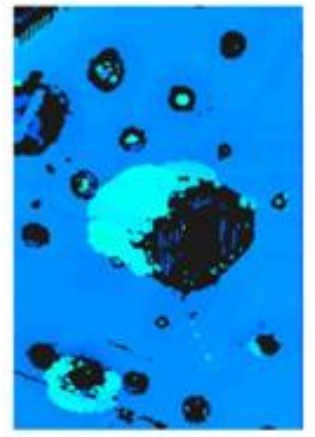

(a)

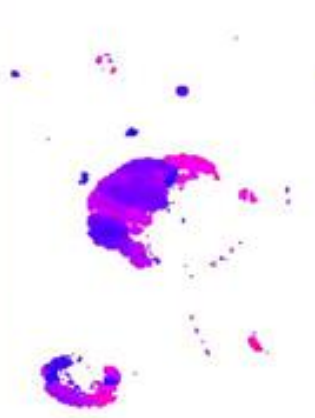

(b)

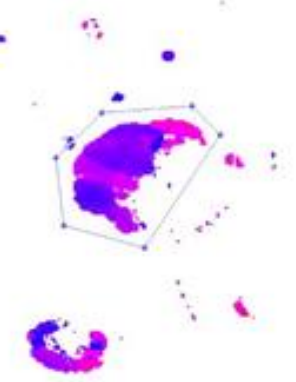

(c)

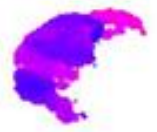

(d)

Fig. 3. Selected algorithm steps of ROI extraction

The second stage of the algorithm includes calculating the di erence between the damage areas in both C-Scans and superimposing both input C-Scans with the detected contours onto one figure (Figure 4(c)) based on locations of the centroids of these contours.

The obtained results showed that the proposed algorithm may be helpful in the damage evaluation procedures and the superimposing of two C-Scans with damage contours may facilitate the diagnostic inference process. In particular, this may help in the detection of the exact location of potential damage propagation.

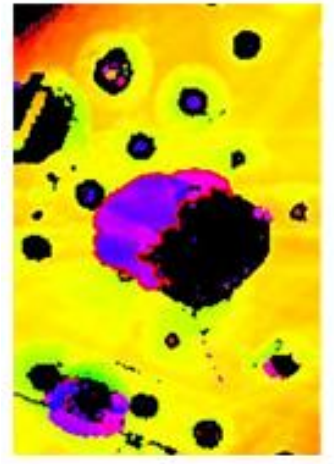

(a)

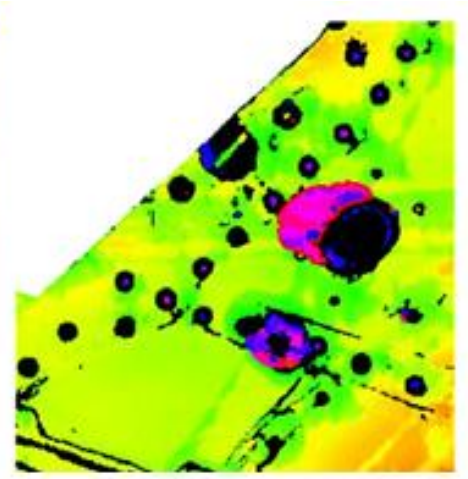

(b)

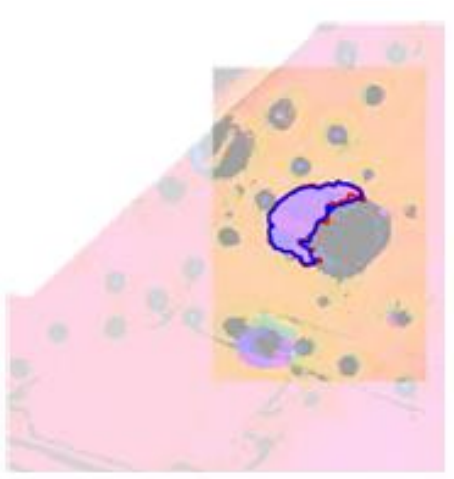

(c)

Fig. 4. Damage contours in the input C-Scans $(a, b)$ and in merged C-Scans (c)

\subsection{D damage reconstruction}

The last study includes 3D reconstruction and visualisation of C-Scans of the composite aircraft panel. Initial results of the tests presented in this section were published in [13]. Three exemplary C-Scan parts of the aircraft element used in this study are presented in Figure 5. 

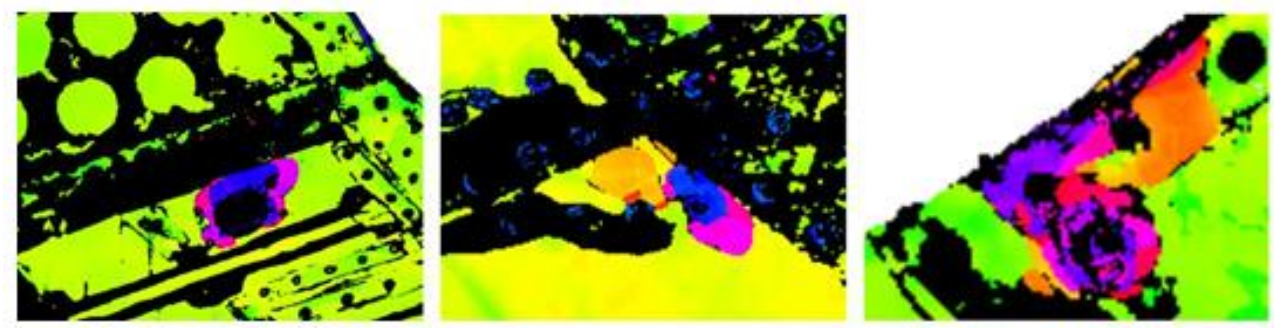

Fig. 5. Parts of the C-Scan of the aircraft panel with detected delaminations

The algorithm can be divided into two steps: damage extraction using the MBP-based segmentation approach and 3D reconstruction with its visualisation. The reconstruction of the extracted damage to a $3 \mathrm{D}$ form is performed in such a way that the values of the $2 \mathrm{D}$ image are distributed to a 3D data array, where each unique value lies on a level (layer) corresponding to this value. Additional rendering is performed for visualisation of damage and background simultaneously, but with di erent transparency. Exemplary views of the obtained 3D visualisations are presented in Figure 6.
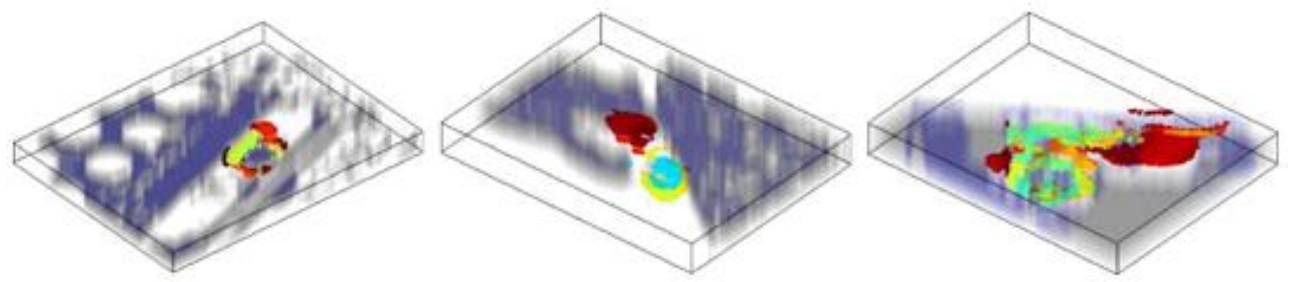

Fig. 6. 3D visualisation of damage sites with backgrounds of the tested aircraft panel

\section{Conclusions}

In this paper, selected algorithms based on image analysis methods were presented that were developed in order to support the ultrasonic-based damage evaluation procedures. Several results were presented that were obtained based on ultrasonic data of the composite aircraft panels with the damage induced during in-service. The presented method for the damage detection is a non-parametric approach, which allows for a quick extraction of damage and its contour, as well as calculation of its size. The method of the damage size monitoring allows for additional comparison of damage detected during separate inspections and visual comparison of both contours. This may also facilitate the detection of the precise location of the damage propagation. It was also found out that the use of the presented 3D recon-struction and visualisation method may simplify interpretation of CScan images. Arbitrary data manipulation in three axes allows for easier evaluation of the damage shape and distri-bution at various depth locations. Distribution of the damage and its directionality as well as $3 \mathrm{D}$ shape may help in inferencing about potential damage root cause. The proposed methods are computationally e cient and can be a useful tool for UT operators. 
This publication is supported within the framework of the Rector's grant no. 10/060/RGJ18/0081 in the area of scientific and development research of the Silesian University of Technology, Poland.

\section{References}

1. R. Wanhill, Milestone Case Histories in Aircraft Structural Integrity, $\mathbf{1}$ of Comprehensive Structural Integrity, pp. 61-72, (2003)

2. D.K. Hsu, in Non-Destructive Evaluation (NDE) of Polymer Matrix Composites, edited by V.M. Karbhari, (Woodhead Publishing Series in Com-posites Science and Engineering, pp. 397-422, 2013)

3. E. Bozzi, G. Cavaccini, M. Chimenti, M. Di Bono, O. Salvetti, Pattern Recognition and Image Analysis 17, 545 (2007)

4. A. Momtaz, A. Sadr, International Journal of Simulation, Systems, Science and Technology 10, 34 (2009)

5. S. Li, A. Poudel, T.P. Chu, Informatica 37, 359 (2013)

6. S. Samanta, D. Datta, Imaging of impacted composite armours using data clustering, in Proc. of the 18th World Conference on Nondestructive Testing (Durban, South Africa, 2012)

7. S. Li, A. Poudel, T.P. Chu, An Image Enhancement Technique for Ultrasonic NDE of CFRP Panels, in Proc. of the 21st Annual Research Symposium \& Spring Conference (Dallas, TX, 2012)

8. K. Dragan, M. Stefaniuk, P. Synaszko, Composites Theory and Practice 12, 105 (2012b)

9. S. Li, T.P. Chu, Ultrasonic 3D reconstruction of CFRP panel delaminations, in Fall Conference \& Quality Testing Show 2012 (Orlando, FL, 2012)

10. A. Wronkowicz, K. Dragan, M. Dziendzikowski, M. Chalimoniuk, C. Sbarufatti, 3D Reconstruction of Ultrasonic B-Scans for Nondestructive Testing of Composites, in Proc. of the International Conference on Computer Vision and Graphics, edited by L.J. Chmielewski, A. Datta, R. Kozera, K. Wojciechowski (Springer, 2016)

11. A. Wronkowicz, G. Mihaylov, K. Dragan, A. Timofiejczuk, Advances in Engineering Software (submitted) (2018b)

12. A. Wronkowicz, K. Dragan, Composites Theory and Practice 16, 154 (2016)

13. A. Wronkowicz, A. Katunin, K. Dragan, International Journal of Image, Graphics and Signal Processing 7, 1 (2015) 